\title{
De novo ALX4 variant detected in child with non-syndromic craniosynostosis
}

\author{
C.S. Fonteles ${ }^{1 \oplus 凶}$, R.H. Finnell ${ }^{2 \oplus}$, Y. Lei ${ }^{3 \oplus}$, M.E. Zurita-Jimenez ${ }^{4 \oplus}$, A.J. Monteiro ${ }^{5 \oplus}$, \\ T.M. George ${ }^{6 \dagger}$, and R.J. Harshbarger ${ }^{7 \oplus}$
}

${ }^{1}$ Programa de Pós-graduação em Odontologia, Faculdade de Farmácia, Odontologia e Enfermagem, Universidade Federal do Ceará, Fortaleza, CE, Brasil ${ }^{2}$ Center for Precision Environmental Health, Departments of Molecular and Cellular Biology, Molecular and Human Genetics and Medicine, Baylor College of Medicine, Houston, TX, USA ${ }^{3}$ Center for Precision Environmental Health, Department of Molecular and Cellular Biology, Baylor College of Medicine, Houston, TX, USA

${ }^{4}$ Dell Pediatric Research Institute, Dell Medical School, The University of Texas at Austin, Austin, TX, USA

${ }^{5}$ Departamento de Estatística e Matemática Aplicada, Universidade Federal do Ceará, Fortaleza, CE, Brasil ${ }^{6}$ Plastic Surgery, Craniofacial Team at the Dell Children's Medical Center of Central Texas, Department of Neurosurgery, Dell Medical School, The University of Texas at Austin, Austin, TX, USA ${ }^{7}$ Plastic Surgery, Craniofacial Team at the Dell Children's Medical Center of Central Texas, Department of Pediatrics, Dell Medical School, The University of Texas at Austin, Austin, TX, USA

\begin{abstract}
Current understanding of the genetic factors contributing to the etiology of non-syndromic craniosynostosis (NSC) remains scarce. The present work investigated the presence of variants in ALX4, EFNA4, and TWIST1 genes in children with NSC to verify if variants within these genes may contribute to the occurrence of these abnormal phenotypes. A total of 101 children (aged $45.07 \pm 40.94$ months) with NSC participated in this cross-sectional study. Parents and siblings of the probands were invited to participate. Medical and family history of craniosynostosis were documented. Biological samples were collected to obtain genomic DNA. Coding exons of human TWIST1, ALX4, and EFNA4 genes were amplified by polymerase chain reaction and Sanger sequenced. Five missense variants were identified in $A L X 4$ in children with bilateral coronal, sagittal, and metopic synostosis. A de novo ALX4 variant, c.799G >A: p.Ala267Thr, was identified in a proband with sagittal synostosis. Three missense variants were identified in the EFNA4 gene in children with metopic and sagittal synostosis. A TWIST1 variant occurred in a child with unilateral coronal synostosis. Variants were predicted to be among the $0.1 \%(T W I S T 1$, c.380C $>$ A: p. Ala127Glu $)$ and 1\% (ALX4, c.769C > T: p.Arg257Cys, c.799G >A: p.Ala267Thr, c.929G >A: p.Gly310Asp; EFNA4, c.178C > T: p. His60Tyr, C.283A > G: p.Lys95Glu, c.349C >A: Pro117Thr) most deleterious variants in the human genome. With the exception of $A L X 4, c .799 G>A$ : p.Ala267Thr, all other variants were present in at least one non-affected family member, suggesting incomplete penetrance. Thus, these variants may contribute to the development of craniosynostosis, and should not be discarded as potential candidate genes in the diagnosis of this condition.
\end{abstract}

Key words: Congenital abnormalities; Mutation; Missense mutation; DNA; Saliva; Cranial sutures

\section{Introduction}

During early childhood, the fusion of cranial sutures must follow a pre-programmed physiological time table in order to allow adequate growth of the developing brain (1). Different signaling pathways that enable continuance of suture patency may be disrupted, resulting in craniosynostosis. With a prevalence of approximately 3.1-6.4 per 10,000 live births, craniosynostosis can affect one or more cranial sutures, occurring as an isolated clinical entity or in association with a group of craniofacial syndromes. A recent survey in the Netherlands showed an overall rise in the prevalence of craniosynostosis and synostosis of the sagittal and metopic sutures (2).

In 2015, Twigg and Wilkie (3) published a review reporting a total of 57 genetic variants identified in at least

Correspondence: C.S. Fonteles: <cfontele@ufc.br>

This paper is dedicated to the memory of the late Dr. Timothy M. George, whose devotion to the care of children with craniofacial disorders was unsurpassed. 
2 patients with craniosynostosis. Recently, Goos and Mathijssen (4) found 39 novel genes that cause craniosynostosis. Twenty-two of these variants were reported in at least 2 individuals, rendering a total of at least 79 craniosynostosis variants. These variants may interfere with essential processes in the preservation of suture patency during growth and development, such as osteogenic differentiation, maintenance of mesoderm-neural crest lineage boundary, and bone remodeling (5). Specific missense variants in fibroblast growth factor receptor genes (FGFR) 1, 2, and 3 are frequently linked to early suture fusion in various autosomal dominant syndromes that express craniosynostosis, including: Apert, Crouzon, Muenke, Pfeiffer, and Jackson-Weiss syndromes (6). However, variants in these genes have not been associated with non-syndromic craniosynostosis (NSC).

Previous studies identified variants in ALX homeobox 4 (ALX4: NM_021926.3; MIM \#605420) and Homo sapiens ephrin A4 (EFNA4: NM_005227; MIM \#601380) in association with non-syndromic forms of craniosynostosis $(7,8)$. In addition, new variants associated with NSC were identified in the Homo sapiens Twist family bHLH transcription factor 1 (TWIST1: MIM \#601622; NM_00 0474.3), and other genes known to cause syndromic forms of these malformations were also identified in affected individuals $(9,10)$. In spite of these previous findings, ALX4 and EFNA4 genes are not usually considered potential candidate genes when investigating genetic causes of NSC, and earlier work failed to demonstrate the presence of mutations associated with syndromic craniosynostosis within the exons of TWIST1 in suture cells of individuals with single suture craniosynostosis (11). In light of this information, we aimed to evaluate if children with different subtypes of NSC expressed variants in these genes. We have investigated patient medical histories, and have Sanger DNA-sequenced all coding regions pertaining to ALX4, EFNA4, and TWIST1 genes in a population of 101 children diagnosed with NSC. Samples from the children's mother and/or father and siblings were also investigated when available.

\section{Material and Methods}

\section{Patient recruitment and study design}

This cross-sectional study followed the Strengthening the Reporting of Observational Studies (STROBE) guidelines. Children of all ages (both sexes) presenting to the Dell Children's Craniofacial and Reconstructive Plastic Surgery Center at the Dell Children's Medical Center of Central Texas (USA) from May 2015 to August 2016, with a diagnosis of craniosynostosis were screened for participation. Diagnosis of craniosynostosis was based on physical examination by a craniofacial team, medical history review, cranial computed tomography, and magnetic resonance imaging scans. Postoperative surgical reports were also taken into consideration when making

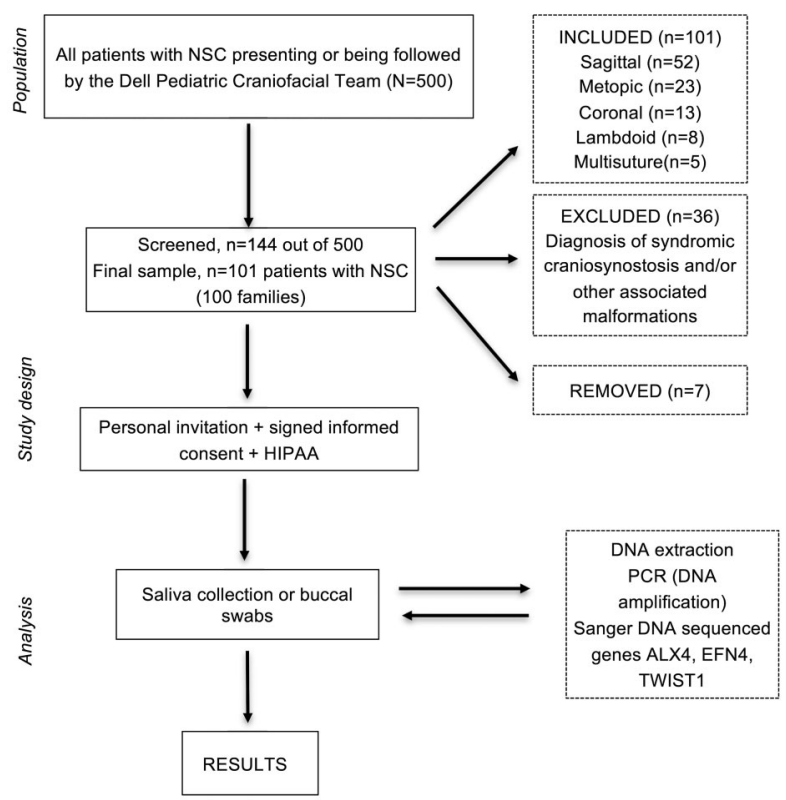

Figure 1. Flowchart showing study design, patient recruitment, and final study sample. NSC: non-syndromic craniosynostosis; HIPAA: Health Insurance Portability and Accountability Act.

the definitive diagnosis. Craniosynostosis syndromes were identified and excluded from the study (Figure 1). Patient screening and recruitment and sample collection occurred during the child's preoperative evaluation for surgical correction of craniosynostosis or during postsurgical follow-up visits. Sample size was determined by the number of families and patients who agreed to participate within the time of patient recruitment (convenience sample). Biological parents and siblings of affected children were also invited to participate in the study. Information regarding medical/family history, ethnicity, sex, and age was provided by the parents and by accessing patients' electronic files. Patient and family enrollment in the study occurred only after the written informed consent forms were appropriately signed. Signature of HIPAA (Health Insurance Portability and Accountability Act) forms allowed access to the patient's electronic files. This study was approved by the Seton Hospital Institutional Review Board (Austin, TX, USA).

\section{Collection of biological samples}

Biological samples were collected from children and their families to obtain genomic DNA. For saliva collection, a minimum of $2 \mathrm{~mL}$ of saliva was collected from children using the Oragene Discover kit (DNA Genotek Inc., Canada). Buccal cell sampling utilizing Isohelix DNA buccal swabs (SK-2 swabs; Cell Projects Ltd., UK) was the alternative sampling method when there was no cooperation for saliva collection. These kits provided a safe and reliable method of obtaining genomic DNA for 
future sequencing analysis, allowing sample storage and transportation to the research lab at room temperature. Genomic DNA was subsequently extracted through an established protocol using the Puregene DNA Purification kit (Qiagen, USA) for saliva and the Isohelix DNA Isolation kit for buccal cell samples.

\section{DNA sequencing}

All coding exons of human TWIST1 (NM_000474.3), ALX4 (NM_021926), and EFNA4 (NM_005227) genes were amplified by polymerase chain reaction (PCR) $\left(\mathrm{T} 100^{\mathrm{TM}}\right.$ Thermal Cycler, Bio-Rad Laboratories, USA). Primer3 was used to design primers. Primers covered intro/splice sites. We included at least 50 nt flanking the splice sites of target exon. Primer sequences and PCR conditions are available upon request. The PCR products were sequenced using the BigDye ${ }^{\mathbb{R}}$ Terminator v3.1 Cycle Sequencing kit (Applied Biosystems, USA), in addition to either a specific forward or reverse primer. Samples were processed in the 3730 DNA Analyzer (Thermo Fisher Scientific, USA). DNA sequences were aligned and analyzed using the Mutation Surveyor V4.0.7 (Softgenetics, USA). The obtained sequences were mapped using Genome Reference Consortium Human Build 38 (GRCh38/hg3). Previously reported variants in human TWIST1, ALX4, and EFNA4 were determined based on their presence in four public databases: dbSNP (http://www.ncbi.nlm.nih. gov/snp), the 1000 Genome Project (http://www.1000 genomes.org), the NHLBI GO Exome Sequencing Project (https://esp.gs.washington.edu/drupal/), and the Exome Aggregation Consortium (ExAC) Browser (http://exac. broadinstitute.org/). Additional PCR and sequencing analyses were performed to confirm the presence of the identified rare variants $(<1 \%)$. The outcome of rare missense variants on the coded protein was predicted in silico using the PolyPhen-2 (Polymorphism Phenotyping version 2.1.0; http://genetics.bwh.harvard.edu/pph/), SIFT (Sorting Intolerant From Tolerant; http://sift.jcvi.org/), and CADD (Combined Annotation Dependent Depletion; https://cadd.gs.washington.edu/score) programs (12). Pathogenicity of these variables was also assessed by applying the American College of Medical Genetics and Genomics Standards and Guidelines (ACMG) (13).

\section{Data analysis}

The outcome measure was presence of at least one TWIST1, ALX4, or EFNA4 variant described as being probably damaging (PolyPhen,) and damaging (SIFT) in children with craniosynostosis. The main exposure was defined as having a confirmed diagnosis of NSC. In order to control for potential confounders, biological samples were collected from parents and siblings. Presence of coinciding genotypes between the proband and at least one other family member that did not express craniosynostosis led to the understanding that the proband's genotype was not pathogenic. Association of sex, ethnic- ity, craniosynostosis subtypes, family history of craniosynostosis, presence/absence of variants, and affected genes was performed using chi-squared test. The Fisher exact test was used to compare the frequency of the rare $(\mathrm{MAF}<0.001)$ ALX4 and EFNA4 variants and controls in the Genome Aggregation Database (gnomAD). Significance was set at $\mathrm{P}<0.05$.

\section{Results}

\section{Population, ethnicity, and family history}

A total of 101 children (aged $45.07 \pm 40.94$ months) with NSC, including 43 parent-offspring trios, 49 motheroffspring pairs, and 2 father-offspring pairs participated in the present study. Six children were enrolled in the study without the participation of other family members. A maleto-female ratio of $2.74: 1$ was observed within this population, (males: $n=74,73.3 \%$; females: $n=27,26.7 \%$ ). Siblings of affected individuals from 26 families also agreed to participate in the study. Fifteen patients (14.9\%) within the study population reported a positive family history of craniosynostosis.

Craniosynostosis of the sagittal suture $(51.5 \%, n=52)$ was the most frequent subtype, followed by synostosis of the metopic $(22.8 \%, n=23)$, unilateral coronal $(12.9 \%$, $\mathrm{n}=13)$, and lambdoid $(7.9 \%, \mathrm{n}=8)$ sutures (Figure 1). Five children $(5.0 \%)$ expressed a multi-suture synostosis, including bilateral coronal synostosis $(n=3)$ and involvement of the lambdoid and sagittal sutures $(n=2)$. Demographic distribution of patients in our study was as follows: Hispanic $(48.5 \%, n=49)$ and White children $(42.6 \%, n=43)$ were more frequently affected than children of Asian $(5.0 \%, n=5)$ and African $(4.0 \%, n=4)$ descent. There was no significant association between craniosynostosis subtype and sex $(P=0.184)$ or ethnicity $(P=0.494)$. Children with a family history of craniosynostosis were affected by the isolated forms of sagittal, metopic, or unilateral coronal craniosynostosis. Families with children affected by unilateral lambdoid $(n=8)$ or multi-suture craniosynostosis $(n=5$, bilateral coronal craniosynostosis included) did not report having a family history of craniosynostosis.

\section{DNA sequencing analysis}

Five different missense variants were identified in the ALX4 gene (c.769C > T: p.Arg257Cys; c.799G >A: p.Ala267Thr; c.929G >A: p.Gly310Asp; c.304C >T: p. Pro102Ser; and c.104G > C: p.Arg35Thr) (Supplementary Table S1). These variants were present in children with the following phenotypes: bilateral coronal ( $A L X 4$, c.769C > T: p.Arg257Cys), sagittal (ALX4, c.799G >A: p.Ala267Thr, c.929G >A: p.Gly310Asp), and metopic synostosis $(A L X 4$, c.304C $>$ T: p.Pro102Ser, c. $104 G>C$ : p.Arg35Thr). Variant $A L X 4$, c.799G $>$ A: p.Ala267Thr was identified as a de novo mutation affecting the HOX domain of the $A L X 4$ protein. It was not identified in the proband's parents or siblings (Figures 2 and 3 ). Three missense 
FATHER
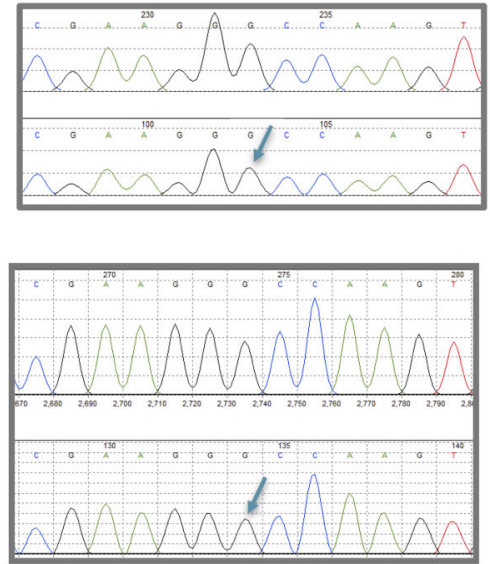

SIBLING 1
MOTHER
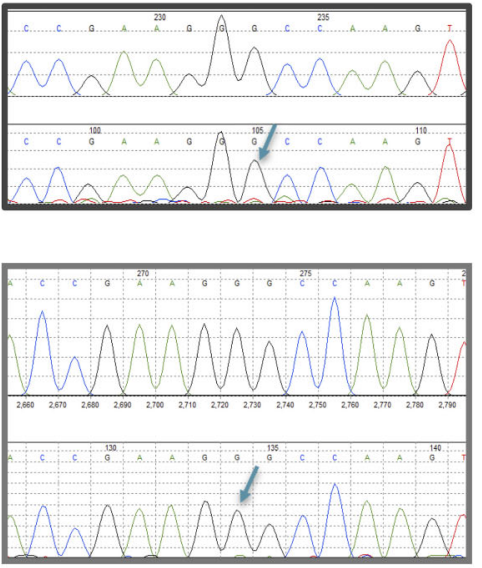

SIBLING 2

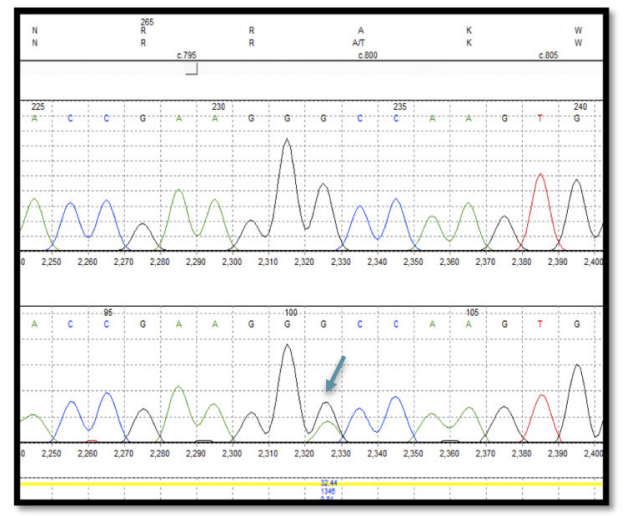

PROBAND

Ala267Thr

Figure 2. Chromatogram showing presence of de novo ALX4 variant in proband and absence in family members.

variants were identified in EFNA4 in children with metopic (c.178C > T: p.His60Tyr and C.283A > G: p.Lys95Glu) and sagittal synostosis, whereas a TWIST1 missense variant (c.380C > A: p.Ala127Glu) affected a child with unilateral coronal synostosis. One child with metopic synostosis expressed concomitant variants in the $A L X 4$ (c.304C $>\mathrm{T}$ : p.Pro102Ser and c.104G > C: p.Arg35Thr) and EFNA4 (C.283A > G: p.Lys95Glu) (Supplementary Table S1) genes. The variant $A L X 4$ (c.304C > T: p.Pro102Ser) was predicted as being tolerated and benign by SIFT and Polyphen databases, respectively. Types of variants ( $A L X 4$, EFN4, or TWIST1) were not significantly associated with specific craniosynostosis subtypes $(P=0.221)$. The presence/absence of variants was not significantly associated with craniosynostosis subtypes $(P=0.708)$, ethnicity $(P=0.414)$, or sex $(P=0.605)$.

\section{Discussion}

While the etiology of non-syndromic forms of craniosynostosis remains unclear, efforts in recent years have increased our basic understanding of several gene variants associated with these malformations. In the present study, we have investigated the presence of variants in genes previously associated with NSC phenotypes $(7,8,14)$. Despite earlier and recent evidence (15), the ALX4 and EFNA4 genes are frequently not considered candidate genes when investigating the genetic causes of NSC. In addition, TWIST1 has been shown to cause both syndromic and non-syndromic forms of synostosis, but it is more frequently investigated in the presence of coronal and sagittal forms of these malformations. We have therefore chosen to screen these three genes for possible 

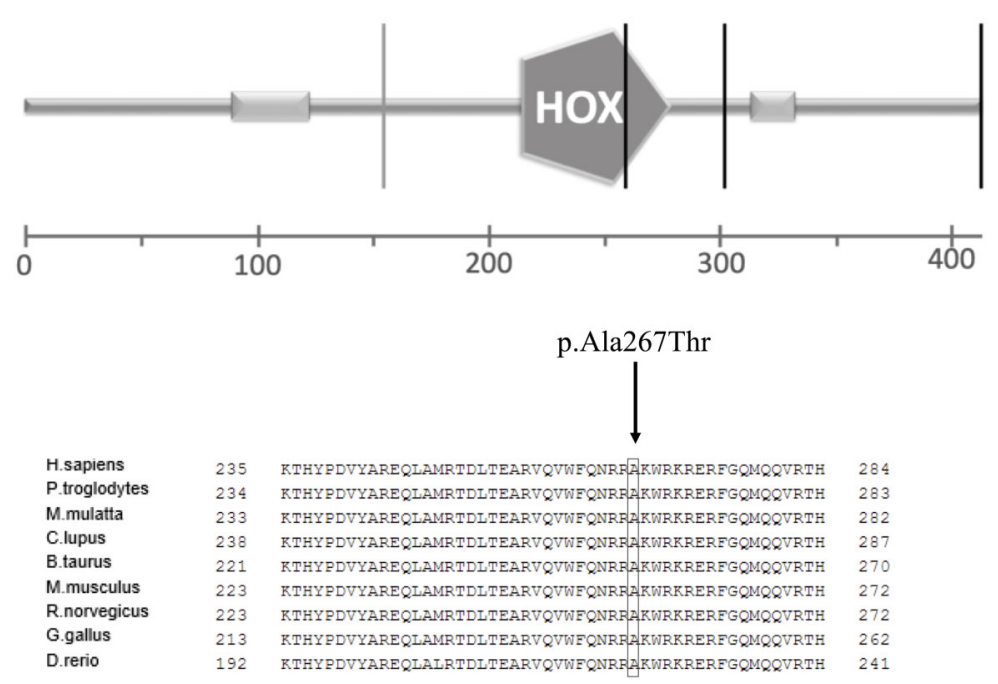

Figure 3. HOX domain within Homo sapiens Homeobox protein aristaless-like 4 and sequence alignment between species.

pathogenic variants in a population of children with different forms of non-syndromic single- and multi-suture synostosis. In our cohort, nine different variants affecting these genes were identified in probands with metopic (ALX4, EFNA4), sagittal (ALX4, EFNA4), and unilateral or bilateral coronal (TWIST1, ALX4) craniosynostosis. These variants were not identified in patients with craniosynostosis of the lambdoid suture or with multi-suture synostosis involving other sutures.

An earlier cohort of 203 patients identified five $A L X 4$ variants in individuals with either sagittal or multi-suture synostosis with the involvement of the sagittal suture (8). Functional analysis of three previously reported variants revealed gain-of-function effect for two of these variants (Val7Phe and Lys211Glu). In the present work, five missense variants were identified in the $A L X 4$ gene. Two of these variants (c.304C > T: p.Pro102Ser and c.104G > C: p.Arg35Thr) are not rare in the general population and are predicted as benign by SIFT and PolyPhen, while the other three variants (c.769C $>$ T: p.Arg257Cys; c.799G $>$ A: p.Ala267Thr; and c.929G $>$ A: p.Gly310Asp) constitute novel missense variants in the $A L X 4$ gene. $A L X 4$ variant Gly310Asp showed conflicting results, being tolerated with a score of 0.1 (Sift), probably damaging in PolyPhen (score of 0.454), and a CAAD of 24.8, thus being classified as likely pathogenic (ACMG). The patient's mother did not present the same variant, and the father was not available for DNA testing. Two of the identified variations in amino acid substitutions (Arg257Cys and Ala267Thr) were predicted by SIFT as being damaging to protein function, with a score of 0 and confirmed to be probably damaging with a PolyPhen score of 1. A white female with bilateral coronal synostosis harbored the Arg257Cys variant, which was also present in the child's unaffected mother. However, the child's unaffected sibling expressed a different genotype, whereas the father did not enroll in the study. This finding suggested that this variant was not the sole cause of craniosynostosis in this patient, but may show incomplete penetrance, or predispose the individual to craniosynostosis, but not cause it $(16,17)$.

We identified a de novo ALX4 variant c.799G $>A$ (Ala267Thr) in a proband with sagittal craniosynostosis. The child's parents and siblings did not harbor this variant and had a normal phenotype, suggesting that this variant is pathogenic. The role of $A L X 4$ in calvarial development appears to be associated with osteogenic differentiation and modulation of osteoblast function, working within a consortium of transcription factors alongside MSX2, subsequent to lineage commitment of the mesenchymal progenitor cells $(18,19)$. Further, heterozygous loss-offunction variants in $A L X 4$ and $M S X 2$ genes produce similar phenotypic outcomes, including an enlarged parietal foramen (20). It has been demonstrated in $A L X 4$ knockout mice that inactivation of this gene adversely impacts the transcription of Spp1, resulting in a reduction in the expression of FGFR1 and FGFR2 within the parietal and frontal bones (18). We do know that FGFR1 and FGFR2 signaling pathways fulfill specific regulatory functions on suture osteogenesis by controlling the proliferation of osteogenic stem cells (FGFR2) and by regulating cell differentiation (FGFR1) (21). On the other hand, Spp1 acts as a key regulator in the bone remodeling process by directly binding to apatite crystals and inhibiting mineralization, in addition to exerting a role in osteoclast recruitment, stimulating adhesion, migration, and bone resorption by these cells (22). Thus, the Ala267Thr variant may create an imbalanced signal expression downstream of $A L X 4$, and the early closure of the sagittal suture in this Hispanic patient may be the negative consequence of such a signaling error. This amino acid substitution affects the HOX domain of the $A L X 4$ protein that lies between 
positions 214 and 276. Reported $A L X 4$ variants affecting this domain cause parietal foramina 2 (PFM2, MIM \# 609597) (23), which is also a clinical feature of PotockiShaffer syndrome (MIM \# 601224), a rare contiguous gene deletion on chromosome $11 \mathrm{p} 11.2$ that involves the ALX4 gene (24).

In the present work, $3 / 101$ individuals presented with variants in the EFNA4 gene. Two of these patients had a diagnosis of metopic craniosynostosis, while one patient had sagittal craniosynostosis. Interestingly, the male proband with metopic synostosis that carried protein change Lis95Glu also carried two additional $A L X 4$ variants (c.304C > T: p.Pro102Ser; c.104G > C: p.Arg35Thr). The EFNA4 variant $(c .283 A>G)$ was predicted as probably damaging (PolyPhen score 0.981) and likely pathogenic (ACMG), with a CADD of 23.8. Concomitant variants should be viewed with caution, since their concurrent presence may alter their disease-causing potential (25). Merrill and colleagues described EFNA4 variants that were associated with metopic (c.178C $>\mathrm{T}$ : p.His60Tyr) and sagittal (c.349C $>$ A: p.Pro117Thr) craniosynostosis in patients with non-syndromic unilateral coronal craniosynostosis (14). The variant c. $178 \mathrm{C}>\mathrm{T}$ : p.His60Tyr was also identified by Clarke et al. in two patients with single suture craniosynostosis (coronal and sagittal) (7). Merrill et al. (14) demonstrated that existent boundary defects between neural crest and mesoderm cell populations in the junction between parietal and frontal bones lead to early closure of the coronal suture in Twist $1^{+/}$mouse embryos. These Twist1 mutants showed an increase in MSX2 expression, with lower tissue distribution of EphrinA4, perhaps explaining the importance of EFNA4 in the etiology of craniosynostosis of the coronal suture. It was also demonstrated that these missense variants (c.349C $>A$ : p.Pro117Thr and c.178C $>$ T: p.His60Tyr) cause loss of function and compromise binding of Ephrin-A4 to the EphA7 receptor. High affinity Eph/ephrin interactions are essential for neural crest cell migration and maintenance of tissue boundary by mediating attractive and repulsive effects between cells (26). Failure in tissue boundary integrity may be one of the mechanistic failures generated by pathogenic EFNA4 variants. The sagittal suture is also formed at the mesoderm-neural crest interface. Loss of tissue boundary may adversely affect the metopic suture, which is exclusively of neural crest origin (27).

Previously, TWIST1 gene variants were identified in patients with Saethre-Chotzen syndrome and syndromic craniosynostosis (28-30). Evidence suggests that variants within this gene are not considered as a causative or contributing factor in non-syndromic, single-suture craniosynostosis (11). Nevertheless, TWIST1 variants are frequently associated with coronal craniosynostosis, and this type of premature suture closure is more commonly of genetic origin (9). In 2006, Kress et al. (31) reported a rare variant (c.602C $>$ T, p.Ser201Tyr) in a child with sagittal
NSC and his unaffected mother. The authors proposed that this variant was non-pathogenic. Seto et al. (16) identified variants in the TWIST box region of TWIST1 in two different patients presenting with isolated sagittal synostosis (c.563C > T: p.Ser188Leu) and unilateral coronal synostosis (c.556G $>$ A: p.Ala186Thr). In the present work, a TWIST1 variant was found in a Hispanic child with unilateral coronal NSC and a positive family history of this malformation. This variant was in the top $0.1 \%$ of deleterious variants in the human genome, being also a pathogenic variant according to other databases. A sample from the child's unaffected sibling allowed detection of the same variant. We believe that c.380C $>$ A: p. Ala127Glu could be a variant with incomplete penetrance or one that results in variable expressivity, as previously proposed by Seto et al. (16). The possibility of mosaicism must also be considered, since this condition was previously identified for the TWIST1 gene (32). Furthermore, a TWIST1 variant within this location (c.380C $>\mathrm{T}$ ) with a substitution of alanine by valine was previously reported in a patient with Saethre-Chotzen syndrome (33). Hence, variants within this region are likely damaging, emphasizing the need to sequence the TWIST1 gene in patients with coronal craniosynostosis.

The present study had limitations. First, the study was limited to screening only the ALX4, EFNA4, and TWIST1 genes. Previous work has identified variants within other genes in patients with non-syndromic craniosynostosis. Some of these genes include: BMP2, SMAD6, BBS9, MEGF8, SCARF2, FBN1, IGF1R, ATR, ERF, FAM20C, TGFBR2, FGFR1, TCF12, IFT122, IL11RA, MASP1, MEGF8, POR, RAB23, RECQL4, SKI, ALPL, FLNA, HUWE1, IDUA, IFT122, IRX5, KAT6A, KMT2D, and LRP5 $(7,10,25,34,35)$. The sample size was also considered a limitation. The identified variants showed very low frequency in a given population; therefore, we were unable to demonstrate statistical association between these variants and craniosynostosis subtypes. We had difficulties recruiting parent-offspring trios, as not all parents agreed to participate or only one parent (usually the child's mother) was present for medical appointments at which time a sample could be collected. Moreover, many families resided in distant communities and return consultation visits were scheduled only after our recruitment period had ended.

In the present work, the $A L X 4$ gene presented the highest frequency of variants in patients with NSC (5/101), followed by the EFNA4 (3/101) and TWIST 1 (1/101) genes. It is also important to mention that, with the exception of the $A L X 4$ variants c. $304 \mathrm{C}>\mathrm{T}$ (gnomAD frequency of $78.070 / 175.982$ ) and c. $104 \mathrm{G}>\mathrm{C}$ (gnomAD frequency of $121.117 / 244.066$ ) that are relatively common, all other variants were less frequent alleles, featuring among the top $1 \%$ of deleterious variants in the human genome. Based on the current literature and databases, these 
variants are likely pathogenic and may play a contributing role in the development of craniosynostosis. Future population-based studies should help clarify this matter. These results suggested that $A L X 4$ and EFNA4 genes should not be discarded as potential candidate genes to be used for panel testing in the diagnosis of NSC along with other genes (10). Also, the addition of the TWIST1 gene in these panels may be of interest when investigating genetic causes of coronal NSC. The ALX4 c.799G $>$ A (Ala267Thr) is a de novo variant affecting the HOX domain of the $A L X 4$ protein in a proband with premature closure of the sagittal suture. This finding suggested $A L X 4$ as a gene possibly involved in NSC.

\section{References}

1. Fonteles CS, Finnell RH, George TM, Harshbarger RJ. Craniosynostosis: current conceptions and misconceptions. AIMS Genetics 2016; 3: 99-129, doi: 10.3934/genet.2016. 1.99 .

2. Cornelissen $M$, den Ottelander $B$, Rizopoulos $D$, van der Hulst R, van der Molen AM, van der Horst C, et al. Increase of prevalence of craniosynostosis. J Craniomaxillofac Surg 2016; 44: 1273-1279, doi: 10.1016/j.jcms.2016.07.007.

3. Twigg SRF, Wilkie AOM. A genetic-pathophysiological framework for craniosynostosis. Am J Hum Genet 2015; 97: 359-377, doi: 10.1016/j.ajhg.2015.07.006.

4. Goos JAC, Mathijssen IMJ. Genetic causes of craniosynostosis: an update. Mol Syndromol 2019; 10: 6-23, doi: 10.1159/000492266.

5. Wu X, Gu Y. Signaling mechanisms underlying genetic pathophysiology of craniosynostosis. Int J Biol Sci 2019; 15: 298-311, doi: 10.7150/ijbs.29183.

6. Passos-Bueno MR, Sertié AL, Jehee FS, Fanganiello $R$, Yeh E. Genetics of craniosynostosis: genes, syndromes, mutations and genotype-phenotype correlations. Front Oral Biol 2008; 12: 107-143, doi: 10.1159/000115035.

7. Clarke CM, Fok VT, Gustafson JA, Smyth MD, Timms AE, Frazar $C D$, et al. Single suture craniosynostosis: identification of rare variants in genes associated with syndromic forms. Am J Med Genet A 2018; 176: 290-300, doi: 10.10 02/ajmg.a.38540.

8. Yagnik G, Ghuman A, Kim S, Stevens CG, Kimonis V, Stoler $\mathrm{J}$, et al. ALX4 gain-of-function mutations in nonsyndromic craniosynostosis. Hum Mutat 2012; 33: 1626-1629, doi: 10.1002/humu.22166.

9. Ko JM, Jeong SY, Yang JA, Park DH, Yoon SH. Molecular genetic analysis of TWIST1 and FGFR3 genes in Korean patients with coronal synostosis: identification of three novel TWIST1 mutations. Plast Reconstr Surg 2012; 129: 814e821e, doi: 10.1097/PRS.0b013e31824a2dda.

10. Timberlake AT, Furey CG, Choi J, Nelson-Williams C, Yale Center for Genome Analysis, Loring $E$, et al. De novo mutations in inhibitors of Wnt, BMP, and Ras/ERK signaling pathways in non-syndromic midline craniosynostosis. Proc Natl Acad Sci USA 2017; 114: E7341-E7347, doi: 10.1073/ pnas. 1709255114.

11. Anderson PJ, Cox TC, Roscioli T, Elakis G, Smithers L, David DJ, et al. Somatic FGFR and TWIST mutations are

\section{Supplementary Material}

Click to view [pdf].

\section{Acknowledgments}

This study was funded by a Research Grant Award from the American Society of Maxillofacial Surgeons to Dr. Raymond J. Harshbarger in 2013. The authors acknowledge the postdoctoral funding provided by the National Council for Scientific and Technological Development (CNPq, Brazil) on behalf of Dr. Cristiane Sá Roriz Fonteles.

not a common cause of isolated nonsyndromic single suture craniosynostosis. J Craniofac Surg 2007; 18: 312-314, doi: 10.1097/scs.0b013e31802d6e76.

12. Rentzsch P, Witten D, Cooper GM, Shendure J, Kircher M. CADD: predicting the deleteriousness of variants throughout the human genome. Nucleic Acids Res 2019; 8; 47: D886D894, doi: 10.1093/nar/gky1016.

13. Richards S, Aziz N, Bale S, Bick D, Das S, Gastier-Foster J, et al. Standards and guidelines for the interpretation of sequence variants: a joint consensus recommendation of the American College of Medical Genetics and Genomics and the Association for Molecular Pathology. Genet Med 2015; 17: 405-424, doi: 10.1038/gim.2015.30.

14. Merrill AE, Bochukova EG, Brugger SM, Ishii M, Pilz DT, Wall SA, et al. Cell mixing at a neural crest-mesoderm boundary and deficient ephrin-Eph signaling in the pathogenesis of craniosynostosis. Hum Mol Genet 2006; 15: 1319-1328, doi: 10.1093/hmg/ddl052.

15. Sewda A, White SR, Erazo M, Hao K, García-Fructuoso G, Fernández-Rodriguez I, et al. Nonsyndromic craniosynostosis: novel coding variants. Pediatr Res 2019; 85: 463-468, doi: 10.1038/s41390-019-0274-2.

16. Seto ML, Hing AV, Chang J, Hu M, Kapp-Simon KA, Patel $\mathrm{PK}$, et al. Isolated sagittal and coronal craniosynostosis associated with TWIST box mutations. Am J Med Genet A 2007; 143A: 678-686, doi: 10.1002/ajmg.a.31630.

17. Johnson D, Wall SA, Mann S, Wilkie AO. A novel mutation, Ala315Ser, in FGFR2: a gene-environment interaction leading to craniosynostosis? Eur J Hum Genet 2000; 8: 571-577, doi: 10.1038/sj.ejhg.5200499.

18. Antonopoulou I, Mavrogiannis LA, Wilkie AO, Morriss-Kay GM. Alx4 and Msx2 play phenotypically similar and additive roles in skull vault differentiation. J Anat 2004; 204: 487499, doi: 10.1111/j.0021-8782.2004.00304.x.

19. Stains JP, Civitelli R. Genomic approaches to identifying transcriptional regulators of osteoblast differentiation. Genome Biol 2003; 4: 222, doi: 10.1186/gb-2003-47-222.

20. Mavrogiannis LA, Taylor IB, Davies SJ, Ramos FJ, Olivares $\mathrm{JL}$, Wilkie AO. Enlarged parietal foramina caused by mutations in the homeobox genes ALX4 and MSX2: from genotype to phenotype. Eur J Hum Genet 2006; 14: 151-158, doi: 10.1038/sj.ejhg.5201526. 
21. Iseki S, Wilkie AO, Morriss-Kay GM. Fgfr1 and Fgfr2 have distinct differentiation- and proliferation-related roles in the developing mouse skull vault. Development 1999; 126: 5611-5620, doi: 10.1242/dev.126.24.5611.

22. Kahles F, Findeisen HM, Bruemmer D. Osteopontin: A novel regulator at the cross roads of inflammation, obesity and diabetes. Mol Metab 2014; 3: 384-393, doi: 10.1016/j. molmet.2014.03.004.

23. Wuyts W, Cleiren E, Homfray T, Rasore-Quartino A, Vanhoenacker F, Van Hul W. The ALX4 homeobox gene is mutated in patients with ossification defects of the skull (foramina parietalia permagna, OMIM 168500). J Med Genet 2000; 37: 916-920, doi: 10.1136/jmg.37.12.916.

24. Wu YQ, Badano JL, McCaskill C, Vogel H, Potocki L, Shaffer LG. Haploinsufficiency of ALX4 as a potential cause of parietal foramina in the $11 \mathrm{p} 11.2$ contiguous gene-deletion syndrome. Am J Hum Genet 2000; 67: 1327-1332, doi: 10.1016/S0002-9297(07)62963-2.

25. Timberlake AT, Choi J, Zaidi S, Lu Q, Nelson-Williams C, Brooks ED, et al. Two locus inheritance of non-syndromic midline craniosynostosis via rare SMAD6 and common BMP2 alleles. Elife 2016; 5: e20125, doi: 10.7554/eLife.20125.

26. Pasquale EB. Eph receptor signalling casts a wide net on cell behaviour. Nat Rev Mol Cell Biol 2005; 6: 462-475, doi: 10.1038/nrm1662.

27. Jiang X, Iseki S, Maxson RE, Sucov HM, Morriss-Kay GM. Tissue origins and interactions in the mammalian skull vault. Dev Biol 2002; 241: 106-116, doi: 10.1006/dbio.2001.0487.

28. el Ghouzzi V, Le Merrer M, Perrin-Schmitt F, Lajeunie E, Benit $P$, Renier D, et al. Mutations of the TWIST gene in the Saethre-Chotzen syndrome. Nat Genet 1997; 15: 42-46, doi: 10.1038/ng0197-42.
29. Howard TD, Paznekas WA, Green ED, Chiang LC, Ma N, de Luna RIO, et al. Mutations in TWIST, a basic helix-loop-helix transcription factor, in Saethre-Chotzen syndrome. Nat Genet 1997; 15: 36-41, doi: 10.1038/ng0197-36.

30. Johnson D, Horsley SW, Moloney DM, Oldridge M, Twigg SR, Walsh S, et al. A comprehensive screen for TWIST mutations in patients with craniosynostosis identifies a new microdeletion syndrome of chromosome band 7p21.1. Am J Hum Genet 1998; 63: 1282-1293, doi: 10.1086/302122.

31. Kress W, Schropp C, Lieb G, Petersen B, Büsse-Ratzka M, Kunz J, et al. Saethre-Chotzen syndrome caused by TWIST 1 gene mutations: functional differentiation from Muenke coronal synostosis syndrome. Eur J Hum Genet 2006; 14 : 39-48, doi: 10.1038/sj.ejhg.5201507.

32. Apostolopoulou D, Kaxira OS, Hatzaki A, Panagopoulos KP, Alexandrou K, Stratoudakis A, et al. Genetic analysis of syndromic and nonsyndromic patients with craniosynostosis identifies novel mutations in the TWIST1 and EFNB1 genes. Cleft Palate Craniofac J 2018; 55: 1092-1102, doi: $10.1177 /$ 1055665618760412.

33. Gripp $\mathrm{KW}$, Zackai EH, Stolle CA. Mutations in the human TWIST gene. Hum Mutat 2000; 15: 150-155, doi: 10.1002/ (SICl)1098-1004(200002)15:2<150::AID-HUMU3 > 3.0.CO; 2-D.

34. Justice CM, Yagnik G, Kim Y, Peter I, Jabs EW, Erazo M, et al. A genome-wide association study identifies susceptibility loci for nonsyndromic sagittal craniosynostosis near BMP2 and within BBS9. Nat Genet 2012; 44: 1360-1364, doi: 10.1038/ng.2463.

35. Wilkie AOM, Johnson D, Wall SA. Clinical genetics of craniosynostosis. Curr Opin Pediatr 2017; 29: 622-628, doi: 10.1097/MOP.0000000000000542. 